\title{
Lista actualizada de las lagartijas de Ecuador con comentarios acerca de su diversidad
}

\section{Omar Torres-Carvajal ${ }^{1}$}

\author{
${ }^{1}$ Escuela de Biología, Pontificia Universidad Católica del Ecuador, \\ Avenida 12 de Octubre y Roca, Apartado 17-01-2184, Quito, Ecuador \\ omartorcar@gmail.com
}

Recibido: 20, 05, 2011; aprobado: 29, 07, 2011

\begin{abstract}
RESUMEN.- Se presenta una lista actualizada de las especies de lagartijas de Ecuador que incluye 25 adiciones y una omisión a la última lista publicada en papel. Un breve análisis de diversidad y endemismo indica que, a excepción de Galápagos, la costa y estribaciones occidentales de los Andes albergan la mayor diversidad y endemismo de lagartijas en Ecuador. Una lista de especies permanentemente actualizada, junto con información biológica, fotos y mapas de distribución estará disponible en línea a través del portal FaunaWebEcuador. Según las tendencias descritas en este trabajo, se estima que la presente lista continuará creciendo y modificándose a una tasa acelerada durante los próximos años.
\end{abstract}

PALABRAS CLAVE: Diversidad, Ecuador, FaunaWebEcuador, Lagartijas, Squamata.

ABSTRACT.- An updated checklist of lizards from Ecuador including 25 addi-
tions and one omission to the last checklist published on paper is presented. A brief
analysis indicates that, except for Galápagos, the highest diversity and endemism
of lizards in Ecuador occurs in the Pacific coast and western Andean slopes. A
permanently updated checklist including biological information, photographs and
maps will be available online at FaunaWebEcuador. According to the tendencies
described here, the current checklist will continue to expand and change during the
coming years.

KEY WORDS: Diversity, Ecuador, FaunaWebEcuador, Lizards, Squamata. 


\section{ANTECEDENTES}

Ha pasado ya una década desde la última publicación en papel de una lista de lagartijas de Ecuador (1), y más de un siglo desde que en su catálogo de las lagartijas del Museo Británico de Historia Natural, George A. Boulenger incluyó a gran parte de la diversidad de lagartijas ecuatorianas conocida hasta entonces (2). Entre las fechas de publicación de estos trabajos surgieron otras listas o catálogos en papel, algunos de ellos con claves de identificación, de lagartijas sudamericanas $(3,4,5)$ o específicamente de lagartijas ecuatorianas $(4,6,7)$.

La razón por la que se han publicado cuatro versiones de la lista de lagartijas de Ecuador, más o menos una por década, es que cada versión dura poco tiempo antes de desactualizarse. Hay dos procesos que explican esto. Primero, nuevas especies para la ciencia o para el Ecuador son descubiertas con cierta frecuencia; y segundo, gracias a los avances en biología sistemática de lagartijas, nuevos reordenamientos taxonómicos, que implican cambios en las listas, también son publicados frecuentemente.

Con el "Internet" como recurso para divulgar información, publicar listas de especies en papel podría considerarse como un método de divulgación obsoleto e innecesariamente costoso. Es cierto, y por ello el Museo de Zoología QCAZ de la Pontificia Universidad Católica del
Ecuador se ha encargado de mantener y actualizar una lista en línea de especies de reptiles de Ecuador desde el año 2000. Sin embargo, la actualización de esta lista se detuvo hace más de un año como parte de un proceso de cambio a través del cual el Museo QCAZ publicará en línea una enciclopedia de los vertebrados de Ecuador llamada FaunaWebEcuador ${ }^{1}$. En ella no solamente se listarán a las especies de lagartijas ecuatorianas, sino que también habrá información acerca de su identificación, taxonomía, historia natural, distribución, coloración, así como fotos, figuras, mapas y claves.

La principal razón para presentar nuevamente una lista de lagartijas ecuatorianas es la gran cantidad de cambios que se han dado durante la última década, lo cual refleja no sólo un incremento en el número de investigadores interesados en el estudio de las lagartijas de Ecuador, sino también la falta de conocimiento sobre algo que parecería tan simple como la diversidad en un país tan pequeño, pese a que las primeras publicaciones describiendo lagartijas ecuatorianas se remontan al siglo XVIII.

\section{Adiciones a la lista de lagartijas} del 2001.- Durante la última década se han publicado 20 artículos científicos añadiendo 25 especies a la lista de lagartijas de Ecuador, es decir aumentando el número de especies reconocidas en

${ }^{1}$ http://zoologia.puce.edu.ec/vertebrados/Vertebrata.aspx 
Ecuador en aproximadamente un 13\%. Torres-Carvajal (1) reportó 151 especies (una de ellas ha sido omitida de la presente lista, ver abajo), mientras que la presente lista incluye 175 especies. Las 25 especies añadidas a la lista del 2001 tienen cuatro orígenes distintos:

Especies nuevas para la ciencia $(\mathrm{N}$ $=13$, en orden cronológico): Lepidoblepharis conolepis (8), Echinosaura orcesi (9), Echinosaura brachycephala (10), Stenocercus puyango (11), Thecadactylus solimoensis (12), Enyalioides touzeti (13), Enyalioides rubrigularis (14), Conolophus marthae (15), Anolis anchicayae (16), Anolis lyra (16), Anolis otongae (17), Anolis podocarpus (18), Riama crypta (19).

Nuevos registros para el Ecuador $(\mathrm{N}$ $=6$, en orden cronológico): Polychrus peruvianus (20), Polychrus liogaster (21), Hemidactylus frenatus (22), Anolis heterodermus (23), Anolis ventrimaculatus (Ayala-Varela y Velasco, en preparación), Anolis soinii (Ayala-Varela et al., en prensa).

Especies resucitadas taxonómicamente $(\mathrm{N}=4$, en orden cronológico): Stenocercus angulifer (24); Microlophus indefatigabilis, Microlophus barringtonensis y Microlophus jacobi (25).

Especies omitidas por Torres-Carvajal (1) $(\mathrm{N}=2)$ : Anolis lemniscatus y Kentropyx altamazonica. La primera especie fue omitida por error. En cuanto a $K$. altamazonica, pese a que fue reportada para Ecuador en un catálogo de es- camados del neotrópico (5), esta especie no fue registrada en Ecuador en una monografía detallada del género Kentropyx publicada 22 años más tarde (26). Por esta razón, esta especie fue excluida de la lista de Torres-Carvajal (1). Sin embargo, he confirmado la presencia de $K$. altamazonica en Ecuador con un espécimen depositado en la colección del Museo de Zoología de la PUCE (QCAZ 3725; Provincia Zamora-Chinchipe, destacamento militar Shaime, $1000 \mathrm{~m}$, Lat.: -4.3344, Long.: -78.6807).

\section{Omisiones a la lista de lagartijas} del 2001.- Torres-Carvajal (1) listó a la subespecie Polychrus gutturosus spurellii como una especie, $P$. spurellii, en base a su descripción original (27). Sin embargo, Parker (28) no encontró diferencias entre esta especie y $P$. gutturosus, por lo cual propuso a $P$. spurellii como subespecie de P. gutturosus. Al no existir trabajos científicos que rechacen la propuesta de Parker (28), en la presente lista no se reconoce a $P$. spurellii como una especie válida.

\section{Cambios taxonómicos de la últi-} ma década.- Gracias al rápido avance de la sistemática filogenética, especialmente aquella basada en secuencias de ADN, se han logrado descubrir las relaciones evolutivas de un sinnúmero de clados de lagartijas con representantes en Ecuador. Nuevos árboles filogenéticos han llevado a nuevos reordena- 
mientos taxonómicos, tales como cambios en los nombres de algunos clados asignados a rangos de "familia" o "género" según la taxonomía tradicional (Tabla 1).
Estas divisiones son arbitrarias y de ninguna manera representan la variedad de ecosistemas existentes en Ecuador; sin embargo, la mayoría de especies (67 \%) continentales no ocurren en más de una de estas siete divisiones. El piso altitudi-

Tabla 1. Cambios taxonómicos relacionados con especies de lagartijas presentes en Ecuador. Se presenta el nombre del taxón utilizado en la lista del 2001 de Torres-Carvajal (1), el nombre reconocido actualmente y la(s) referencia(s) correspondiente(s).

\begin{tabular}{ccc}
\hline 2001 & 2011 & Referencia(s) \\
\hline Gekkonidae & Gekkonidae, Phyllodactylidae & $(31,32)$ \\
Neusticurus & y Sphaerodactylidae & \\
Phenacosaurus & Potamites & $(33)$ \\
Prionodactylus & Anolis & $(34)$ \\
Proctoporus & Cercosaura & $(35)$ \\
Tropidurus plica & Riama & $(33)$ \\
Tropidurus flaviceps & Pracentron flaviceps & $(36)$ \\
Tropidurus umbra & Plica umbra & $(36)$ \\
\hline
\end{tabular}

Diversidad y endemismo.- Ya que la mayor parte del territorio ecuatoriano está atravesado de norte a sur por dos cordilleras andinas que separan a la costa del Pacífico de la región amazónica, resulta interesante realizar un análisis simple de la distribución y diversidad de lagartijas dividiendo a los Andes en "pisos altitudinales" de 1000 metros cada uno a ambos lados de los Andes (i.e., Tropical 0-1000 m, Subtropical 1000-2000 m, Templado 2000-3000 m y Altoandino $>3000 \mathrm{~m}$ ). nal con más diversidad (Figura 1) es el Tropical Occidental (59 especies), seguido por el Tropical Oriental (50 especies), Subtropical Occidental (35 especies), Subtropical Oriental (28 especies), Templado Occidental (23 especies), Templado Oriental (16 especies) y Altoandino (9 especies). Todos los pisos bajo los $3000 \mathrm{~m}$ son más diversos al occidente de los Andes, donde se encuentra el 60 $\%$ de las especies continentales de lagartijas. Por otro lado, siguiendo un patrón 
similar al de muchos organismos (29), la diversidad de lagartijas disminuye con la altitud a ambos lados de los Andes ecuatorianos (Figura 1). En Galápagos hay 24 especies de lagartijas, cuatro de las cuales son introducidas (Gonatodes caudiscutatus, Hemidactylus frenatus, Lepidodactylus lugubris, Phyllodactylus reissii) y están presentes también en Ecuador continental.

Las especies de lagartijas de Ecuador pertenecen a ocho clados considerados como "familias" según la taxonomía tradicional. Solamente dos de estos clados contienen aproximadamente el $79 \%$ de las especies, Iguanidae y Gymnophthalmidae, con 88 y 50 especies respectivamente. Les siguen Sphaerodactylidae y Teiidae con 11 especies cada uno, Phyllodactylidae con 10 especies, Gekkonidae con tres especies, y finalmente Anguidae y Scincidae con una especie cada uno (Figura 2).

De las 175 especies reconocidas en el presente trabajo, 70 (40\%) son endémicas para Ecuador. Más de un tercio de este endemismo corresponde a las especies endémicas para Galápagos-seis especies de salamanquesas, 10 de lagartijas de lava, tres de iguanas terrestres y una de iguana marina. De las 50 especies restantes, la mayor parte está dentro de los clados Gymnophthalmidae (23 especies) y Tropidurinae (10 especies) con Riama y Stenocercus como los géneros más representativos respectivamente.
Todos los pisos altitudinales definidos anteriormente contienen especies endémicas, aunque en menor número que Galápagos (Figura 1). Los pisos con más endemismo corresponden al Subtropical Occidental y Templado Occidental (16 especies cada uno), seguidos por el Templado Oriental (13 especies), Tropical Occidental (12 especies), Subtropical Oriental (11 especies), Altoandino (6 especies) y Tropical Oriental (2 especies). De manera similar a los patrones de diversidad descritos arriba, todos los pisos altitudinales bajo los $3000 \mathrm{~m}$ son más diversos al occidente de los Andes, donde se encuentra el $60 \%$ de las especies continentales de lagartijas. Sin embargo, una gran diferencia con los patrones de diversidad es que el endemismo en el piso Tropical Oriental es bastante bajo (Figura 1).

\section{Lista de especies de lagartijas de} Ecuador.- A continuación se listan en orden alfabético por familia, género y especie a las 175 especies de lagartijas de Ecuador reconocidas en el presente trabajo. Las especies endémicas están marcadas con un asterisco. Junto al nombre de cada especie se indica su autor y año de descripción (en paréntesis si el nombre genérico original es distinto al actual); las referencias correspondientes no están listadas en la literatura citada, pero están disponibles en FaunaWebEcuador o en Torres-Carvajal (1). 


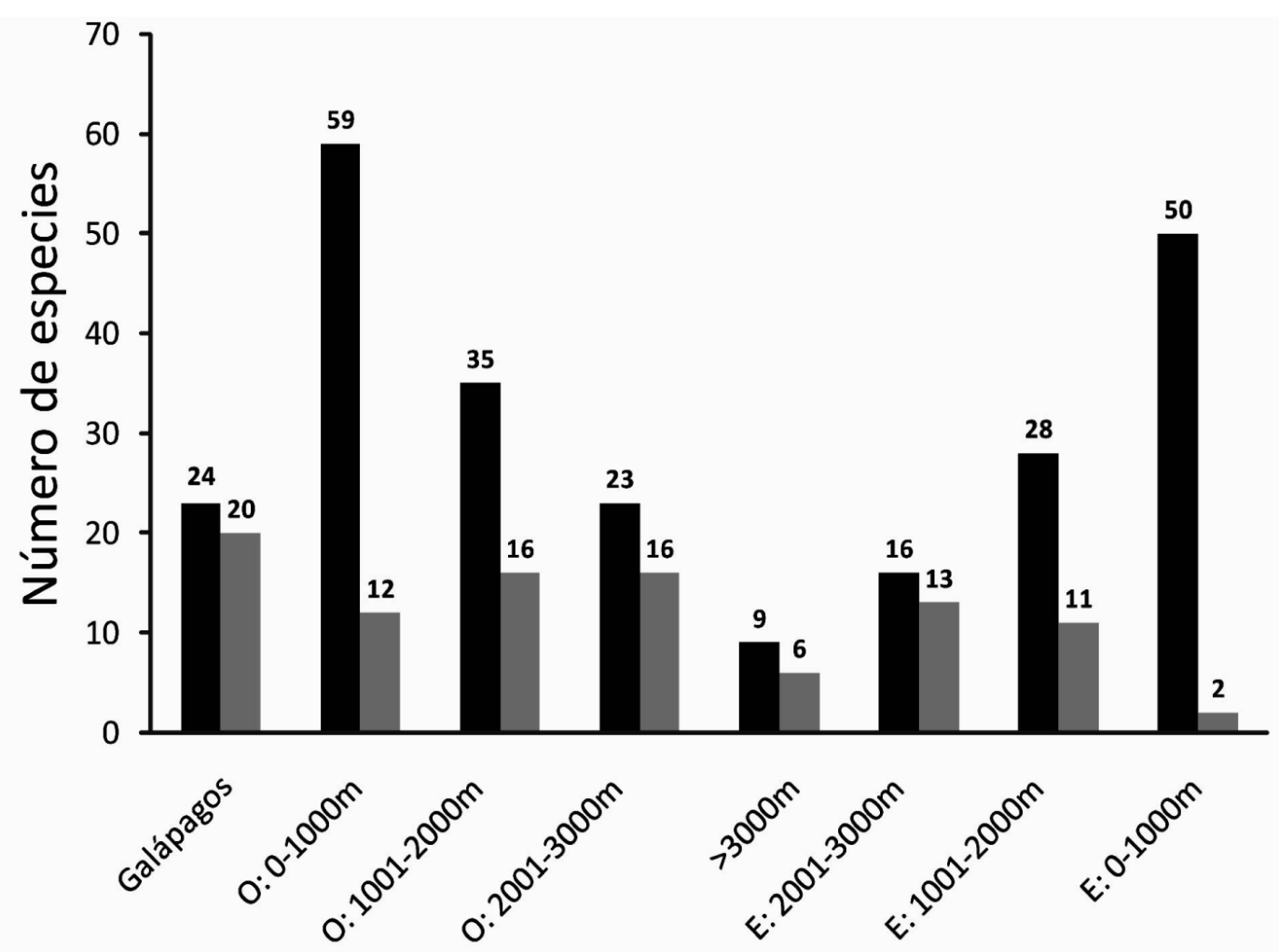

Figura 1. Diversidad y endemismo de lagartijas en Ecuador. Las barras oscuras corresponden al número total de especies por piso altitudinal, mientras que las barras claras corresponden al número de especies endémicas. Sobre cada barra se indica el número absoluto de especies. $\mathrm{O}=$ oeste de los Andes; $\mathrm{E}=$ este de los Andes.

ANGUIDAE (1)

Diploglossus monotropis (Kuhl, 1820)

GEKKONIDAE (3)

Hemidactylus frenatus Schlegel, 1836

Hemidactylus mabouia (Moreau de

Jonnès, 1818)

Lepidodactylus lugubris (Duméril y

Bibron, 1836)
GYMNOPHTHALMIDAE (50)

Alopoglossus angulatus (Linnaeus, 1758)

Alopoglossus atriventris Duellman, 1973

Alopoglossus buckleyi (O'

Shaughnessy, 1881)

Alopoglossus copii Boulenger, 1885

Alopoglossus festae Peracca, 1904

*Anadia petersi Oftedal, 1974

Anadia rhombifera (Günther, 1859)

Arthrosaura reticulata (O' 
Shaughnessy, 1881)

Bachia trisanale (Cope, 1868)

Cercosaura argula (Peters, 1862)

* Cercosaura dicra Uzzell, 1973

Cercosaura manicata (O'Shaughnessy, 1881)

Cercosaura oshaughnessyi Boulenger, 1885

Cercosaura vertebralis (O'

Shaughnessy, 1879)

* Echinosaura brachycephala Köhler et al., 2004

Echinosaura horrida Boulenger, 1890

Echinosaura orcesi Fritts et al., 2002

Euspondylus guentheri (O'

Shaughnessy, 1881)

Euspondylus maculatus Tschudi, 1845

Iphisa elegans Gray, 1851

Leposoma parietale (Cope, 1885)

*Pholidobolus affinis (Peters, 1862)

*Pholidobolus annectens (Parker, 1930)

*Pholidobolus macbrydei Montanucci, 1973

Pholidobolus montium (Peters, 1862)

*Pholidobolus prefrontalis Montanucci, 1973

Potamites cochranae Burt y Burt, 1931

Potamites ecpleopus Cope, 1876

Potamites strangulatus (Cope, 1868)

*Ptychoglossus bilineatus Boulenger, 1890

Ptychoglossus brevifrontalis Boulenger, 1912

Ptychoglossus gorgonae Harris, 1994

* Riama anatoloros Kizirian, 1996

*Riama balneator Kizirian, 1996
*Riama cashcaensis Kizirian y Coloma, 1991

Riama colomaromani Kizirian, 1996

* Riama crypta Sánchez-Pacheco et al., 2011

Riama hyposticta Boulenger, 1902

* Riama labionis Kizirian, 1996

*Riama meleagris Boulenger, 1885

*Riama oculata (O'Shaughnessy, 1879)

*Riama orcesi Kizirian, 1995

* Riama petrorum Kizirian, 1996

* Riama raneyi Kizirian, 1996

Riama simotera (O'Shaughnessy, 1879)

* Riama stigmatoral Kizirian, 1996

* Riama unicolor (Gray, 1858)

* Riama vespertina Kizirian, 1996

* Riama vieta Kizirian, 1996

*Teuchocercus keyi Fritts y Smith, 1969

\section{IGUANIDAE: CORYTOPHANINAE}

(1)

Basiliscus galeritus Duméril y Duméril, 1851

IGUANIDAE: HOPLOCERCINAE (9)

Enyalioides cofanorum Duellman, 1973

Enyalioides heterolepis (Bocourt, 1874)

Enyalioides laticeps (Guichenot, 1855)

Enyalioides microlepis (O'

Shaughnessy, 1881)

Enyalioides oshaughnessyi (Boulenger, 1881)

Enyalioides praestabilis (O'

Shaughnessy, 1881)

*Enyalioides rubrigularis Torres-

Carvajal et al., 2009 
Enyalioides touzeti Torres-Carvajal et al., 2008

Morunasaurus annularis (O'

Shaughnessy, 1881)

IGUANIDAE: IGUANINAE (5)

*Amblyrhynchus cristatus Bell, 1825

*Conolophus marthae Gentile y Snell, 2009

*Conolophus pallidus Heller, 1903

*Conolophus subcristatus (Gray, 1831)

Iguana iguana (Linnaeus, 1758)

IGUANIDAE: POLYCHROTINAE (42)

Anolis aequatorialis Werner, 1894

Anolis anchicayae Poe et al., 2009

Anolis binotatus Peters, 1863

Anolis biporcatus Williams, 1966

*Anolis bitectus Cope, 1864

Anolis bombiceps Cope, 1876

Anolis chloris Boulenger, 1898

Anolis chocorum Williams y Duellman,

1967

Anolis chrysolepis Duméril y Bibron,

1837

* Anolis fasciatus Boulenger, 1885

*Anolis festae Peracca, 1904

Anolis fitchi Williams y Duellman, 1984

Anolis fraseri Günther, 1859

Anolis fuscoauratus D' Orbigny, 1837

Anolis gemmosus O' Shaughnessy,

1875

Anolis gracilipes Boulenger, 1898

Anolis granuliceps Boulenger, 1898

Anolis heterodermus Duméril, 1851

*Anolis lemniscatus Boulenger, 1898
Anolis lynchi Miyata, 1985

Anolis lyra Poe et al., 2009

Anolis maculiventris Boulenger, 1898

*Anolis nigrolineatus Williams, 1965

*Anolis orcesi Lazell, 1969

Anolis ortonii Cope, 1868

*Anolis otongae Ayala-Varela y

Velasco, 2010

*Anolis parilis Williams, 1975

Anolis peraccae Boulenger, 1898

*Anolis podocarpus Ayala-Varela y

Torres-Carvajal, 2010

Anolis princeps Boulenger, 1898

*Anolis proboscis Peters y Orcés-V., 1956

Anolis punctatus Daudin, 1802

Anolis soinii Poe y Yañez-Miranda,

2008

Anolis trachyderma Cope, 1876

Anolis transversalis Duméril y

Duméril, 1851

*Anolis vanzolinii Williams et al., 1996

Anolis ventrimaculatus Boulenger,

1911

Polychrus femoralis Werner, 1910

Polychrus gutturosus Berthold, 1846

Polychrus liogaster Boulenger, 1908

Polychrus marmoratus (Linnaeus, 1758)

Polychrus peruvianus (Noble, 1924)

IGUANIDAE: TROPIDURINAE (31)

*Microlophus albemarlensis (Baur, 1890)

*Microlophus barringtonensis (Baur, 1892)

* Microlophus bivittatus (Peters, 1871)

* Microlophus delanonis (Baur, 1890) 
* Microlophus duncanensis (Baur, 1890)

*Microlophus grayii (Bell, 1843)

* Microlophus habelii (Steindachner, 1876)

*Microlophus indefatigabilis (Baur, 1890)

*Microlophus jacobi (Baur, 1892)

Microlophus occipitalis (Peters, 1871)

*Microlophus pacificus (Steindachner, 1876)

Microlophus peruvianus (Lesson, 1831)

Plica plica (Linnaeus, 1758)

Plica umbra (Linnaeus, 1758)

Stenocercus aculeatus (O'Shaughnessy, 1879)

Stenocercus angel Torres-Carvajal, 2000

* Stenocercus angulifer (Werner, 1901)

*Stenocercus carrioni Parker, 1934

* Stenocercus chota Torres-Carvajal, 2000

* Stenocercus festae (Peracca, 1897)

*Stenocercus guentheri (Boulenger, 1885)

*Stenocercus haenschi (Werner, 1901)

Stenocercus humeralis (Günther, 1859)

Stenocercus iridescens (Günther, 1859)

Stenocercus limitaris Cadle, 1998

*Stenocercus ornatus (Gray, 1845)

Stenocercus puyango Torres-Carvajal, 2005

*Stenocercus rhodomelas (Boulenger, 1899)

*Stenocercus simonsii Boulenger, 1899

* Stenocercus varius Boulenger, 1885

Uracentron flaviceps (Guichenot, 1855)
PHYLLODACTYLIDAE (10)

*Phyllodactylus barringtonensis Van Denburgh, 1912

*Phyllodactylus baurii Garman, 1892

* Phyllodactylus darwini Taylor, 1942

*Phyllodactylus galapagensis Peters, 1869

*Phyllodactylus gilberti Heller, 1903

*Phyllodactylus leei Cope, 1889

* Phyllodactylus pumilus Dixon y Huey, 1970

Phyllodactylus reissii Peters, 1862

Thecadactylus rapicauda (Houttuyn, 1782)

Thecadactylus solimoensis Bergmann y Russell, 2007

SCINCIDAE (1)

Mabuya nigropunctata (Spix, 1825)

SPHAERODACTYLIDAE (11)

Gonatodes caudiscutatus (Günther, 1859)

Gonatodes concinnatus (O'

Shaughnessy, 1881)

Gonatodes humeralis (Guichenot, 1855)

* Lepidoblepharis buchwaldi Werner, 1910

*Lepidoblepharis conolepis AvilaPires, 2001

Lepidoblepharis festae Peracca, 1897

*Lepidoblepharis grandis Miyata, 1985

Lepidoblepharis intermedius

Boulenger, 1914

Lepidoblepharis ruthveni Parker, 1926

Pseudogonatodes guianensis Parker, 
1935

Sphaerodactylus scapularis Boulenger, 1902

TEIIDAE (11)

Ameiva ameiva (Linnaeus, 1758)

Ameiva bridgesii (Cope, 1869)

Ameiva edracantha Bocourt, 1874

* Ameiva orcesi Peters, 1964

Ameiva septemlineata Duméril y

Duméril, 1851

Callopistes flavipunctatus (Duméril y

Bibron, 1839)

Dicrodon guttulatum Duméril y Bibron, 1839

Dracaena guianensis Daudin, 1802
Kentropyx altamazonica (Cope, 1876)

Kentropyx pelviceps Cope, 1868

Tupinambis teguixin (Linnaeus, 1758)

\section{CONCLUSIONES}

Un número considerable de especies nuevas para la ciencia o nuevos registros para el Ecuador han mejorado notablemente nuestro conocimiento sobre la diversidad de lagartijas en este país durante la última década. La tendencia actual sugiere que los datos de diversidad y endemismo presentados en este trabajo cambiarán dramáticamente durante la siguiente década. En general,

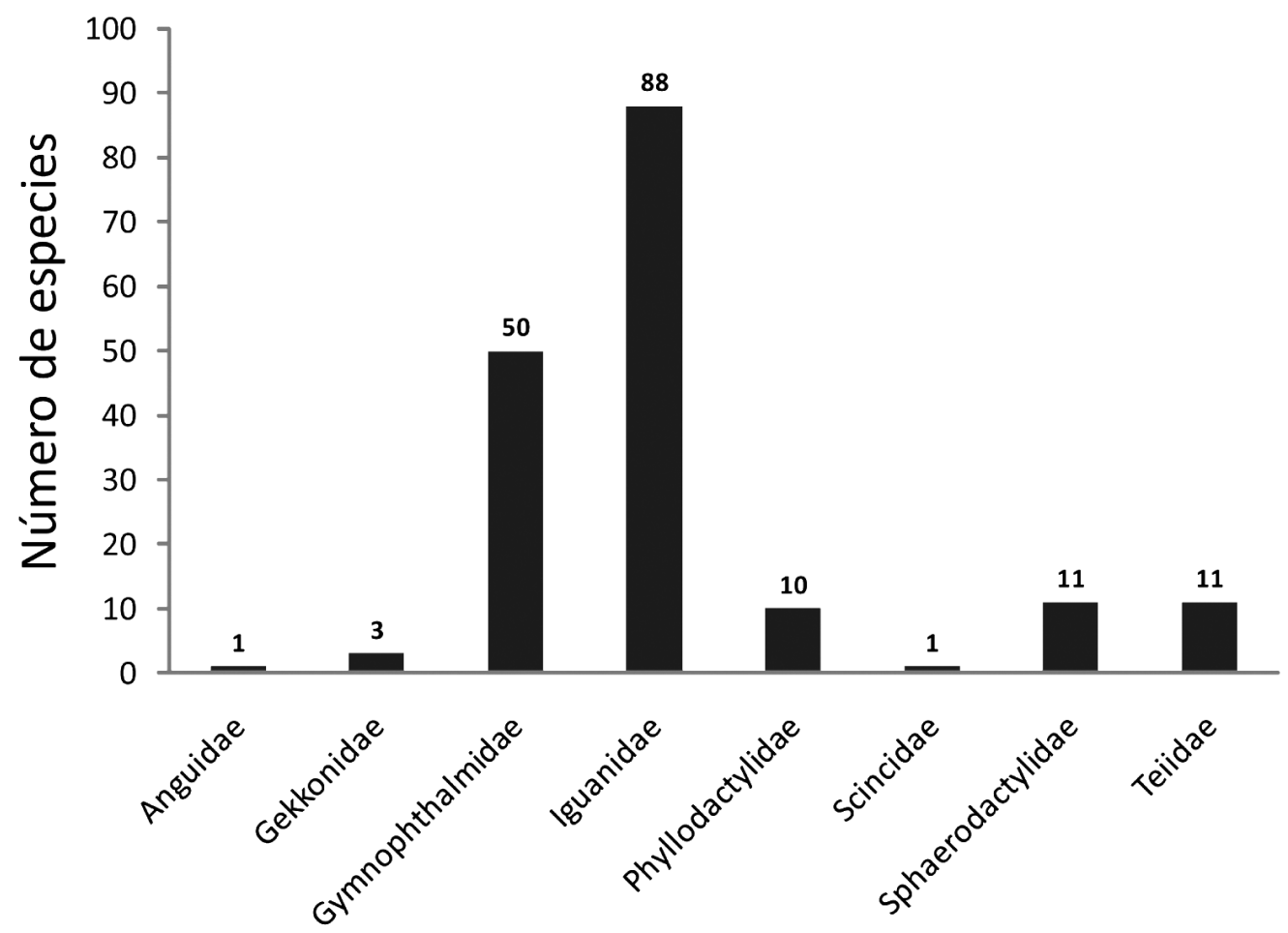

Figura 2. Diversidad taxonómica de lagartijas en Ecuador. Sobre cada barra se indica el número de especies por clado. 


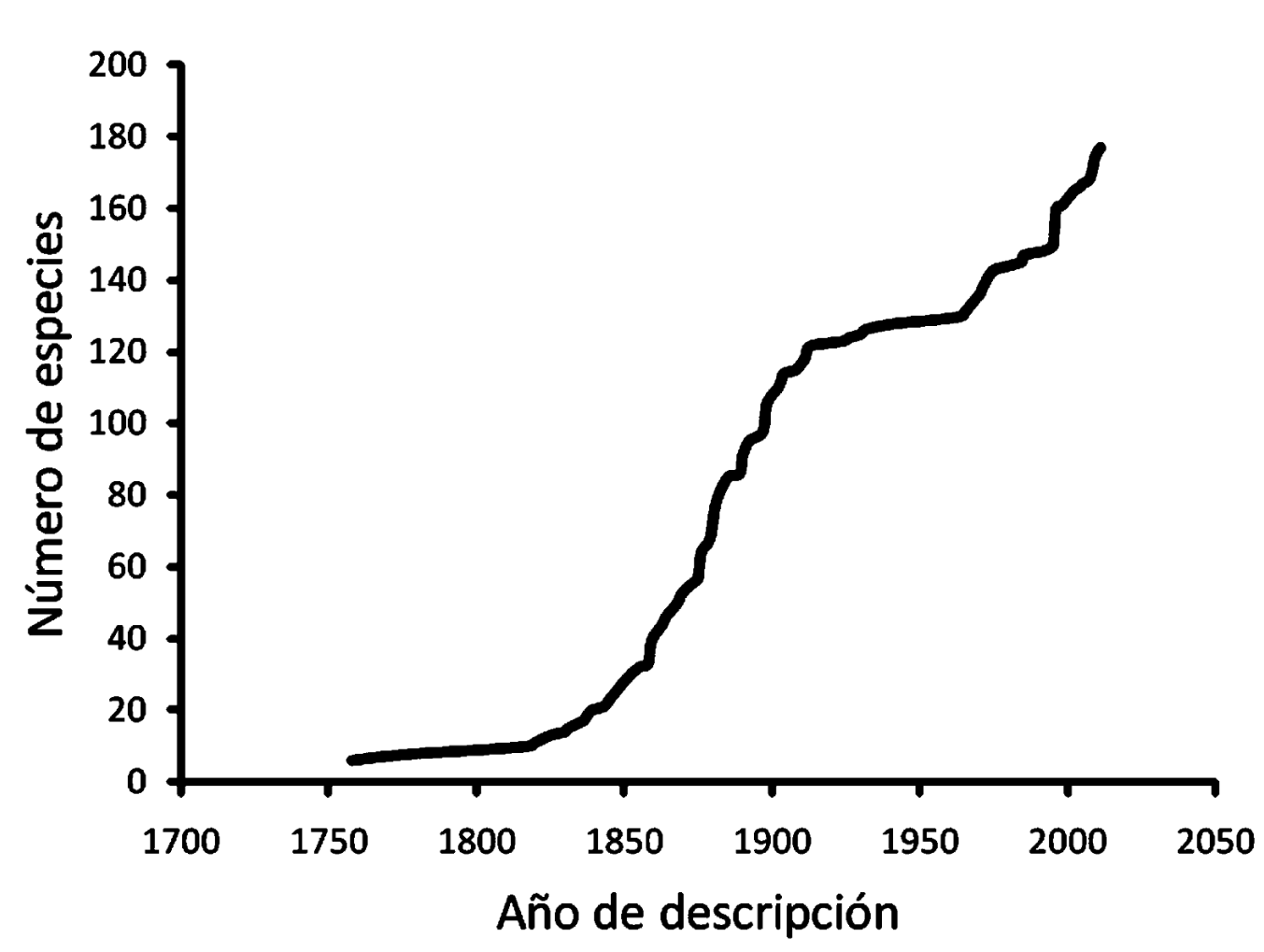

Figura 3. Número de especies de lagartijas de Ecuador descritas por año. No todas las especies fueron descritas en base a especímenes colectados en Ecuador.

la tasa de descubrimiento de especies de lagartijas en Ecuador durante las últimas décadas es similar a aquella de finales del siglo XIX, época marcada por una intensa exploración y estudio de la biodiversidad a nivel mundial (Figura 3 ). Este reciente repunte se da gracias a un incremento en el número de científicos interesados en caracterizar la diversidad de estos animales en Ecuador, y nos deja dos mensajes muy claros que deberían ser reconocidos adecuadamente por todos los actores de nuestra sociedad interesados en el conocimiento y conservación de la biodiversidad:
1) estamos lejos de conocer la totalidad de especies de lagartijas presentes en Ecuador, y 2) es necesario incrementar las colecciones científicas en el campo, especialmente en aquellas áreas donde las colecciones son escasas o nulas. Esta situación no es exclusiva de las lagartijas, es compartida por otros grupos de animales y plantas. Las instituciones y autoridades tanto gubernamentales como privadas tienen el deber moral de apoyar el trabajo (ciencia básica) de cientificos nacionales y extranjeros interesados en el estudio de nuestra biodiversidad. Hoy en día se deben apoyar 
además estudios de sistemática basados en el uso de marcadores moleculares, especialmente secuencias de ADN. Estos estudios, comunes en otros países desde hace décadas, han permitido descubrir un sinnúmero de especies crípticas (dos o más especies imposibles de diferenciar morfológicamente) a nivel mundial (30), y es muy probable que el número de especies crípticas de lagartijas en Ecuador sea considerable.

\section{AGRADECIMIENTOS}

Agradezco a Fernando Ayala, Andrea Rodríguez y Vanessa Aguirre por sus valiosos comentarios a versiones preliminares de este manuscrito. El presente trabajo es el resultado de la actualización permanente de una la lista de lagartijas de Ecuador que ha estado disponible en la página web del Museo de Zoología QCAZ desde el año 2000. Este portal ha progresado significativamente durante los últimos 3 años, y como resultado pronto se publicará la enciclopedia electrónica de vertebrados FaunaWebEcuador, gracias al apoyo económico de JRS Biodiversity Foundation y SENESCYT. Agradezco a todos los integrantes del Museo de Zoología QCAZ involucrados desde hace más de 10 años, inicialmente bajo la dirección de L. A. Coloma, en esta gran iniciativa de difundir gratuitamente información de alta calidad sobre nuestra biodiversidad a través del internet. Finalmente agradezco a Santiago Ron por sus correcciones y comentarios a este manuscrito.

\section{REFERENCIAS \\ BIBLIOGRÁFICAS}

1. TORRES-CARVAJAL, O. 2001. Lizards of Ecuador: checklist, distribution, and systematic references. Smithsonian Herpetological Information Service 131: 1-35.

2. BOULENGER, G. A., Catalogue of the Lizards in the British Museum (Natural History). Second Edition. Three volumes. 1885-1887 (1965), Codicote: Wheldon \& Wesley. xii, 436 p., 32 plates; xiv, 497 p., 24 plates; xii, 575 p., 40 plates.

3. BURT, C. E. \& BURT, M. D. 1933. A preliminary check list of the lizards of South America. Transactions of the Academy of Natural Sciences St. Louis 28(1-2): 1-104.

4. PETERS, J. A. 1967. The Lizards of Ecuador, A Checklist and Key. Proceedings of the United States National Museum 119(3545): 1-49.

5. PETERS, J. A. \& DONOSO-BARROS, R. 1970. Catalogue of the neotropical Squamata: Part II. Lizards and amphisbaenians. United States National Museum Bulletin 297: 1-293.

6. ALMENDÁRIZ, A. 1992. Anfibios y reptiles. Revista Politécnica, Escuela Politécnica Nacional 16(3): 89-162.

7. MIYATA, K. 1982. A check list of 
the amphibians and reptiles of Ecuador with a bibliography of Ecuadorian herpetology. Smithsonian Herpetological Information Service 1982(54): 1-70.

8. AVILA-PIRES, T. C. S. 2001. A new species of Lepidoblepharis (Reptilia: Squamata: Gekkonidae) from Ecuador, with a redescription of Lepidoblepharis grandis Miyata, 1985. Occasional Papers of the Sam Noble Oklahoma Museum of Natural History 11: 1-11.

9. FRITTS, T. H.; ALMENDÁRIZ, A. \& SAMEC, S. 2002. A new species of Echinosaura (Gymnophthalmidae) from Ecuador and Colombia with comments on other members of the genus and Teuchocercus keyi. Journal of Herpetology 36(3): 349355.

10. KÖHLER, G.; BÖHME, W. \& SCHMITZ, A. 2004. A New Species of Echinosaura (Squamata: Gymnophthalmidae) from Ecuador. journal of Herpetology 38(1): 5260.

11. TORRES-CARVAJAL, O. 2005. A new species of iguanian lizard (Stenocercus) from the western lowlands of southern Ecuador and northern Peru. Herpetologica 61(1): 78-85.

12. BERGMANN, P. J. \& RUSSELL, A. P. 2007. Systematics and biogeography of the widespread Neotropical gekkonid genus Thecadactylus
(Squamata), with the description of a new cryptic species. Zoological Journal of the Linnean Society 149: 339-370.

13. TORRES-CARVAJAL, O.; ALMENDÁRIZ, A.; VALENCIA, J.; YÁNEZ-MUÑOZ, M. \& REYES, J. 2008. A new species of Enyalioides (Iguanidae: Hoplocercinae) from southwestern Ecuador. Papéis Avulsos de Zoologia 48(20): 227235.

14. TORRES-CARVAJAL, O.; DE QUEIROZ, K. \& ETHERIDGE, R. 2009. A new species of iguanid lizard (Hoplocercinae, Enyalioides) from southern Ecuador with a key to eastern Ecuadorian Enyalioides. Zookeys 27: 59-71.

15. GENTILE, G. \& SNELL, H. 2009. Conolophus marthae sp.nov. (Squamata, Iguanidae), a new species of land iguana from the Galápagos archipelago. Zootaxa 2201: 1-10.

16. POE, S.; VELASCO, J. A.; MIYATA, K. \& WILLIAMS, E. E. 2009. Descriptions of two nomen nudum species of Anolis lizard from northwestern South America. Breviora 516: 1-16.

17. AYALA-VARELA, F. P. \& VELASCO, J. A. 2010. A new species of dactyloid anole (Squamata: Iguanidae) from the western Andes of Ecuador. Zootaxa 2577: 46-56.

18. AYALA-VARELA, F. P. \& TO- 
RRES-CARVAJAL, O. 2010. A new species of dactyloid anole (Iguanidae, Polychrotinae, Anolis) from the southeastern slopes of the Andes of Ecuador. Zookeys 53: 59-73.

19. SÁNCHEZ-PACHECO, S. J.; KIZIRIAN, D. A. \& NUNES, P. M. 2011. A new species of Riama from Ecuador previously referred to as Riama hyposticta (Boulenger, 1902) (Squamata: Gymnophthalmidae). American Museum Novitates (3719): 1-15.

20. YÁNEZ-MUÑOZ, M.; ORTIZ, F. \& ALTAMIRANO, M. 2006. Reptilia, Polychrotidae, Polychrus peruvianus: New country record, Ecuador. Check List 2(2): 63-64.

21. VIGLE, G. O. 2008. The amphibians and reptiles of the Estación Biológica Jatun Sacha in the lowland rainforest of Amazonian Ecuador: A 20year record. Breviora 514: 1-30.

22. JADIN, R. C.; ALTAMIRANO, M.; YÁNEZ-MUÑOZ, M. \& SMITH, E. N. 2009. First record of the common house gecko (Hemidactylus frenatus) in Ecuador. Applied Herpetology 6: 193-195.

23. TORRES-CARVAJAL, O.; AYALA, F. \& CARVAJAL-CAMPOS, A. 2010. Reptilia, Squamata, Iguanidae, Anolis heterodermus Duméril, 1851: Distribution extension, first record for Ecuador and notes on color variation. Check List 6(1): 189-190.
24. TORRES-CARVAJAL, O. 2007. A taxonomic revision of South American Stenocercus (Squamata: Iguania) lizards. Herpetological Monographs 21: 76-178.

25. BENAVIDES, E.; BAUM, R.; SNELL, H. M.; SNELL, H. L. \& SITES, J. L. 2009. Island biogeography of Galápagos lava lizards (Tropiduridae: Microlophus): species diversity and colonization of the archipelago. Evolution 63(6): 1606-1626.

26. GALLAGHER, D. S. \& DIXON, J. R. 1992. Taxonomic revision of the South American lizard genus Kentropyx Spix (Sauria: Teiidae). Museo Regionale di Scienze Naturali Torino 10(1): 125-171.

27. BOULENGER, G. A. 1914. On a second collection of batrachians and reptiles made by Dr. H. G. F. Spurrell, F.Z.S., in the Choco, Colombia. Proceedings of the Zoological Society of London 1914(3): 813817, 2 plates.

28. PARKER, H. W. 1935. The frogs, lizards, and snakes of British Guiana. Proceedings of the Zoological Society of London 1935: 505-530.

29. STEVENS, G. C. 1992. The Elevational Gradient in Altitudinal Range: An Extension of Rapoport's Latitudinal Rule to Altitude. The American Naturalist 140: 893-911.

30. BICKFORD, D.; LOHMAN, D. J.; SODHI, N. S.; NG, P. K. L.; MEIER, 
R.; WINKER, K.; INGRAM, K. K. \& DAS, I. 2007. Cryptic species as a window on diversity and conservation. Trends in Ecology \& Evolution 22(3): 148-155.

31. GAMBLE, T.; BAUER, A. M.; GREENBAUM, E. \& JACKMAN, T. R. 2008. Evidence for Gondwanan vicariance in an ancient clade of gecko lizards. Journal of Biogeography 35(1): 88-104.

32. GAMBLE, T.; BAUER, A. M.; GREENBAUM, E. \& JACKMAN, T. R. 2008. Out of the blue: a novel, trans-Atlantic clade of geckos (Gekkota, Squamata). Zoologica Scripta 37(4): 355-366.

33. DOAN, T. M. \& CASTOE, T. A. 2005. Phylogenetic taxonomy of the Cercosaurini (Squamata: Gymnophthalmidae), with new genera for species of Neusticurus and Proctoporus. Zoological Journal of the
Linnean Society 143: 405-416.

34. POE, S. 1998. Skull characters and the cladistic relationships of the Hispaniolan dwarf twig Anolis. Herpetological Monographs 12: 192-236.

35. DOAN, T. M. 2003. A new phylogenetic classification for the gymnophthalmid genera Cercosaura, Pantodactylus and Prionodactylus (Reptilia: Squamata). Zoological Journal of the Linnean Society 137: 101-115.

36. FROST, D. R.; RODRIGUES, M. T.; GRANT, T. \& TITUS, T. 2001. Phylogenetics of the lizard genus Tropidurus (Squamata: Tropiduridae: Tropidurinae): Direct optimization, descriptive efficiency, and sensitivity analysis of congruence between molecular data and morphology. Molecular Phylogenetics and Evolution 21(3): 352-371. 